\title{
A DISCRETE ANALOGUE OF THE WEIERSTRASS TRANSFORM ${ }^{1}$
}

EDWARD NORMAN

1. Introduction and definitions. In this note we consider a discrete analogue of the transform

$$
f(x)=\int_{-\infty}^{\infty} e^{-(x-y)^{2} / 4} g(y) d y .^{2}
$$

This transform may be considered as a convolution; accordingly our analogue will take the form

$$
f(n)=\sum_{-\infty}^{\infty} k(n-m) g(m)
$$

where the kernel sequence $k(n)$ is to be appropriately chosen. The choice of $k(n)$ is motivated as follows.

One way in which the kernel $e^{-y^{2} / 4}$ arises in the theory of the convolution transform is by consideration of the class of kernels whose bilateral Laplace transform is of the form

$$
e^{c s^{2}} e^{b s} \prod_{1}^{\infty}\left(1-\frac{s}{a_{k}}\right)^{-1} e^{-s / a_{k}}
$$

When $c=0$ we obtain the class of kernels whose analogue was considered by Pollard and Standish [4] where the kernels formed a subclass of the totally positive sequences. The totally positive sequences can be characterized as sequences having a generating function of the form

$$
e^{a z+b z} z^{m} \prod_{1}^{\infty} \frac{\left(1+\alpha_{j} z\right)\left(1+\beta_{j} z^{-1}\right)}{\left(1-a_{j} z\right)\left(1-b_{j} z^{-1}\right)}
$$

(see [1]). The factor $e^{a z+b z^{-1}}$ in (1.2) corresponds to the factor $e^{c s^{2}}$ in (1.1).

Letting $a=b=v / 2$ we have the familiar expansion

$$
e^{(v / 2)\left(z+z^{-1}\right)}=\sum_{-\infty}^{\infty} I_{m}(v) z^{m}
$$

Received by the editors February 10, 1959 and, in revised form, October 22, 1959.

1 This work is part of a doctoral thesis done under the direction of Professor Harry Pollard at Cornell University.

2 See Hirschman and Widder [3] for the theory of the Weierstrass transform. 
where $I_{m}(v)$ is the modified Bessel coefficient of the first kind.

We take the sequence $\left\{I_{n}(1)\right\}_{n=-\infty}^{\infty}=\left\{I_{n}\right\}_{-\infty}^{\infty}$ as the kernel of our transform

$$
f(n)=\sum_{-\infty}^{\infty} I_{n-m} g(m) .
$$

Before proceeding further we list some elementary properties of the functions $I_{n}(v)$.

$$
\begin{aligned}
I_{n}(v) & =\sum_{0}^{\infty} \frac{(v / 2)^{n+2 s}}{s !(s+n) !}, \quad n=0, \pm 1, \pm \cdots \\
I_{n}(v) & =I_{-n}(v) ; \quad I_{n}(-v)=(-1)^{n} I_{n}(v), \\
\frac{2(1 / 2)^{|n|}}{|n| !} & \leqq I_{n}(1) \leqq \frac{3(1 / 2)^{|n|}}{|n| !}, \\
I_{n}\left(v+v^{\prime}\right) & =\sum_{-\infty}^{\infty} I_{n-m}(v) I_{m}\left(v^{\prime}\right) .
\end{aligned}
$$

If we take $g(m)=2^{|m|}|m| ! /\left(1+m^{2}\right)$ it is clear, by (iii), that (2.13) converges only for the value $n=0$. We therefore define the transform (1.3) only when it converges for all $n$.

Define the operators $\delta$ and $\delta^{-1}$ by the equations

$$
\delta f(n)=f(n-1), \quad \delta^{-1} f(n)=f(n+1) .
$$

Then by analogy with the theory of the Weierstrass transform we may expect (1.3) to be inverted by the operator $e^{-(1 / 2)\left(\delta+\delta^{-1}\right)}$ interpreted as

(1.4) $\left[e^{-(1 / 2)\left(\delta+\delta^{-1}\right)}\right] f(n)=\lim _{t \rightarrow 0+} \sum_{-\infty}^{\infty}(-1)^{m} I_{m} f(n-m) / \Gamma(1+t m)$.

The method of summation adopted in (1.4) is a regular method introduced by Mittag-Leffler (see [2; p. 72]). The motivation for choosing Mittag-Leffler summation as the "natural" method for the inversion operator is indicated in the table below.

\begin{tabular}{l|c|c}
\hline \hline & Continuous & Discrete \\
\hline Kernel & $e^{-y^{2} / 4}$ & $I_{m} 1 / m !$ \\
\hline Convergence factor & $\begin{array}{c}e^{-(1 / t-1) y^{2} / 4} \\
t \rightarrow 1-\end{array}$ & $\begin{array}{c}1 / \Gamma(1+t|m|) \\
t \rightarrow 0+\end{array}$ \\
\hline
\end{tabular}


Unfortunately the operator (1.4) will not serve to invert all convergent transforms (1.3). For example let

$$
g(m)=\left\{\begin{array}{cc}
\frac{2^{m} m !}{m^{\log m}} & m>1, \\
0 & m \leqq 1 .
\end{array}\right.
$$

Then, if $n>0$ say, we have

$$
\begin{aligned}
f(n) & =\sum_{1}^{\infty} I_{n-m} \frac{2^{m} m !}{m^{\log m}}>\sum_{1}^{\infty} \frac{2^{m} m !}{2^{|n-m|}|n-m| ! m^{\log m}} \\
& >\sum_{n}^{\infty} \frac{2^{n} m !}{(m-n) ! m^{\log m}}>\sum_{n}^{\infty} \frac{m^{n}}{m^{\log m}} \cdot
\end{aligned}
$$

If in the last sum above we consider only the term with $m=\left[e^{n / 2}\right]$ we obtain

$$
f(n)>O\left(e^{n^{2} / 4}\right) \quad n \rightarrow+\infty ;
$$

and (1.4) does not converge for any value of $t$.

In view of the above we restrict attention to the class of determining sequences $g(m)$ which satisfy the condition

$$
g(m)=O\left(|m| ! x^{|m|}\right)
$$

as $|m| \rightarrow \infty$, where $x$ is strictly less than 2 . We abbreviate this restriction by writing $g(m) \in X$.

2. The inversion theorem. We first find the order of the transform.

TheoRem 1. If $g(m) \in X$ then $f(n)=\sum_{-\infty}^{\infty} I_{n-m} g(m)$ converges absolutely for all $n$, and

$$
f(n)=O\left(2^{|n|}|n| !(2 / x-1)^{-|n|}\right) \quad|n| \rightarrow \infty .
$$

Proof. The first statement is obvious. To prove the second, note that since the order conditions on $g(m)$ and $I_{m}$ are the same for $m \rightarrow+\infty$ and $m \rightarrow-\infty$ the same is true of $f(n)$. It is therefore sufficient to prove the theorem for $n \rightarrow+\infty$.

$$
|f(n)| \leqq \sum_{-\infty}^{-1} I_{n-m}|g(m)|+\sum_{0}^{\infty} I_{n-m}|g(m)|=S^{\prime}+S .
$$

Since $n>0$ it is clear that $S^{\prime} \leqq$ const. $\sum_{0}^{\infty}(x / 2)^{m} / m^{n} \rightarrow 0$ as $n \rightarrow \infty$. $S \leqq$ const. $2^{n} \sum_{n}^{\infty} \frac{(x / 2)^{m} m !}{(m-n) !}=$ const. $x^{n} \sum_{0}^{\infty}(m+1) \cdots(m+n)(x / 2)^{m}$. 
The formula

$$
\sum_{0}^{\infty}(m+1) \cdots(m+n) y^{m}=n ! /(1-y)^{n+1}, \quad y<1
$$

may be proved by induction. Hence we have

$$
S \leqq \text { const. } \frac{x^{n} n !}{(1-x / 2)^{n}}=\text { const. } 2^{n} n !(2 / x-1)^{-n} .
$$

THEOREM 2. If $g(n) \in X$ and $f(n)=\sum_{-\infty}^{\infty} I_{n-m} g(m)$, then

$$
\lim _{t \rightarrow 0+} \sum_{-\infty}^{\infty}(-1)^{m} I_{m} f(n-m) / \Gamma(1+t|m|)=g(n) .
$$

Proof. There is no loss of generality in taking $n=0$. For any $t>0$

$$
\begin{aligned}
\sum_{-\infty}^{\infty}(-1)^{m} & I_{m} f(-m) / \Gamma(1+t|m|) \\
& =\sum_{m=-\infty}^{\infty}(-1)^{m} I_{m} / \Gamma(1+t|m|) \sum_{r=-\infty}^{\infty} I_{-m-r} g(r) \\
& =\sum_{r=-\infty}^{\infty} g(r) \sum_{m=-\infty}^{\infty}(-1)^{m} I_{-m-r} I_{m} / \Gamma(1+t|m|)=\sum_{r=-\infty}^{\infty} g(r) \mu(r, t) .
\end{aligned}
$$

The interchange of summations is justified by the estimate of Theorem 1.

Since

$$
\mu(0,0)=\sum_{-\infty}^{\infty}(-1)^{m} I_{-m}(1) I_{m}(1)=\sum_{-\infty}^{\infty} I_{-m}(1) I_{m}(-1)=I_{0}(0)=1
$$

(see iv) the theorem will be established if we show

$$
\begin{aligned}
& \lim _{t \rightarrow 0+} \sum_{1}^{\infty} g(r) \mu(r, t)=0, \\
& \lim _{t \rightarrow 0+} \sum_{-\infty}^{-1} g(r) \mu(r, t)=0 .
\end{aligned}
$$

We will prove (2.3); the proof of (2.4) is entirely similar.

As a first step let us replace $1 / \Gamma(1+t|m|)$ by the contour integral

$$
\frac{1}{\Gamma(1+t|m|)}=\frac{1}{2 \pi i} \int_{-\infty}^{(0+)} \frac{e^{u}}{u} u_{-}{ }^{t|m|} d u
$$

where the path of integration is as shown, the circle being of radius greater than one. 


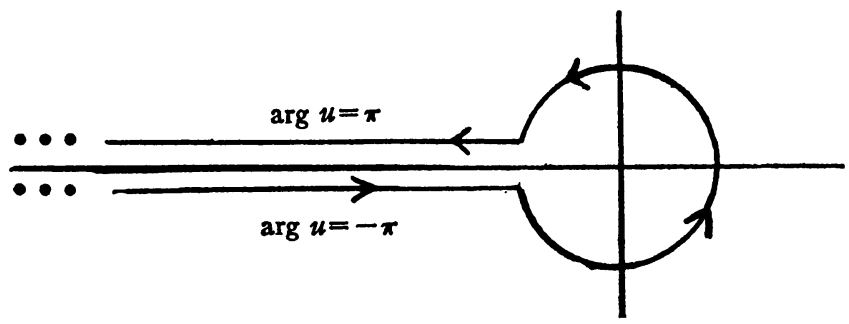

Define the function $K=K(t)=2^{1 / t}(2 / x-1)^{-1 / t}$, so that $K \rightarrow+\infty$ as $t \rightarrow+.^{8}$ For any $t>0$ we have

$$
\begin{aligned}
2 \pi i \sum_{1}^{\infty} g(r) \mu(r, t)= & \sum_{r=1}^{\infty} g(r) \sum_{m=-\infty}^{\infty}(-1)^{m} I_{m} I_{m-r} \int_{-\infty}^{(0+)} \frac{e^{u}}{u} u^{-t|m|} d u \\
= & \sum g(r) \sum(-1)^{m}(\cdots) \int_{C}(\cdots) d u \\
& +\sum g(r) \sum(-1)^{m}(\cdots) \int_{C_{1}}(\cdots) d u \\
& +\sum g(r) \sum(-1)^{m}(\cdots) \int_{C_{2}}(\cdots) d u \\
& =S+S_{1}+S_{2} .
\end{aligned}
$$

The path $C$ runs from $u=-K(\arg u=-\pi)$ round the circle and back to $u=-K(\arg u=\pi), C_{1}$ runs from $-\infty$ to $-K(\arg u=-\pi)$, $C_{2}$ runs from $-K$ to $-\infty(\arg u=\pi)$.

We show now that $\lim _{t \rightarrow 0+} S_{1}=0$; the argument showing $\lim _{t \rightarrow 0+} S_{2}$ $=0$ is the same.

$$
\begin{aligned}
\left|S_{1}\right| & \leqq \sum_{r=1}^{\infty}|g(r)| \sum_{m=-\infty}^{\infty} I_{m} I_{m-r} \int_{C_{1}} \frac{e^{u}}{u} u^{-t|m|} d u \\
& \leqq e^{-K} \sum_{r=1}^{\infty}|g(r)| \sum_{m=-\infty}^{\infty} I_{m} I_{m-r} K^{-t|m|}=e^{-K} \sum_{m=-\infty}^{\infty} I_{m} K^{-t|m|} \sum_{r=1}^{\infty} g(r) I_{m-r} \\
& \leqq e^{-K} \sum_{m=-\infty}^{\infty} I_{m} K^{-t|m|} m ! 2^{|m|}\left(\frac{2}{x}-1\right)^{-|m|}=e^{-K} \sum_{-\infty}^{\infty} I_{m}|m| !=e^{-K} B
\end{aligned}
$$

where $B=\sum_{-\infty}^{\infty} I_{m}|m| !<9$. Therefore $S_{1} \leqq e^{-K} B \rightarrow 0$ as $t \rightarrow 0+$.

8 It is essential to the proof that $K \rightarrow \infty$. This is not the case when $x \leqq 2 / 3$. But in that event the sum (1.4), with $t=0$, converges, and the proof that (1.4) inverts (1.3) is trivial. We therefore assume from now on that $x>2 / 3$. 
The proof has now been reduced to showing that $S \rightarrow 0$ as $t \rightarrow 0+$. To this end we make use of the formula $[5$, p. 441]

$$
I_{m}(1) I_{r-m}(1)=\frac{2}{\pi} \int_{0}^{\pi / 2} I_{r}(2 \cos \theta) \cos (2 m-r) \theta d \theta .
$$

We obtain, setting $u^{-t}=w$,

$$
\begin{aligned}
\frac{\pi}{2} S & =\sum_{r=1}^{\infty} g(r) \sum_{m=-\infty}^{\infty}(-1)^{m} \int_{0}^{\pi / 2} I_{r}(2 \cos \theta) \cos (2 m-r) \theta d \theta \int_{C} \frac{e^{u}}{u} w^{|m|} d u \\
& =\sum_{r=1}^{\infty} g(r) \nu(r, t) .
\end{aligned}
$$

Estimate of $\nu(r, t)$. Since $|w|<1$ along the entire path $C$, we may write

$$
\begin{aligned}
\nu(r, t)= & \int_{C} \frac{e^{u}}{u} \int_{0}^{\pi / 2} I_{r}(2 \cos \theta)\left\{\sum_{m=-\infty}^{\infty}(-1)^{m} w^{m} \cos (2 m-r) \theta\right\} d \theta d u . \\
& \sum_{-\infty}^{\infty}(-1)^{m} w^{|m|} \cos (2 m-r) \theta=\cos (r \theta) \frac{1-w^{2}}{1+2 w \cos 2 \theta+w^{2}} .
\end{aligned}
$$

We now have

$$
\nu(r, t)=\int_{c} \frac{e^{u}}{u}\left(1-w^{2}\right) \int_{0}^{\pi / 2} \frac{I_{r}(2 \cos \theta) \cos r \theta}{1+2 w \cos 2 \theta+w^{2}} d \theta d u .
$$

The integral

$$
Q_{r}(w)=\int_{0}^{\pi / 2} \frac{I_{r}(2 \cos \theta) \cos r \theta}{1+2 w \cos 2 \theta+w^{2}} d \theta
$$

may be estimated as follows.

Replace $I_{r}(2 \cos \theta)$ by the sum (i) and interchange the order of summation and integration to obtain

$$
Q_{r}(w)=\sum_{s=0}^{\infty} \frac{1}{s !(r+s) !} \int_{0}^{\pi / 2} \frac{(\cos ) \theta^{r+2 s} \cos r \theta}{1+2 w \cos 2 \theta+w^{2}} d \theta .
$$

The integral appearing in (2.5) is now estimated by contour integration. It is easily verified that

$$
\int_{0}^{\pi / 2}(\cdots) d \theta=\frac{1}{4} \int_{0}^{2 \pi}(\cdots) d \theta
$$

and so the standard substitution $\cos \theta=1 / 2(z+1 / z), z=e^{i \theta}$ yields 


$$
\begin{aligned}
i 2^{r+2 s+3} \int_{0}^{\pi / 2}(\cdots) d \theta & =\int_{|z|=1} \frac{\left(z^{2}+1\right)^{r+2 s}\left(z^{2 r}+1\right)}{z^{2 r+2 s-1}\left(w z^{4}+\left(1+w^{2}\right) z^{2}+w\right)} d z \\
& =\int_{|z|=1} \rho_{1}(z) d z+\int_{|z|=1} \rho_{2}(z) d z
\end{aligned}
$$

where

$$
\begin{aligned}
& \rho_{1}(z)=\frac{\left(z^{2}+1\right)^{r+2 s}}{z^{2 s-1}\left(w z^{4}+\left(1+w^{2}\right) z^{2}+w\right)} ; \\
& \rho_{2}(z)=\frac{\left(z^{2}+1\right)^{r+2 s}}{z^{2 r+2 s-1}\left(w z^{4}+\left(1+w^{2}\right) z^{2}+w\right)} .
\end{aligned}
$$

Both $\rho_{1}(z)$ and $\rho_{2}(z)$ have poles at $z=0$ and at the zeros of $w z^{4}$ $+\left(1+w^{2}\right) z^{2}+w$, that is, at $z= \pm i w^{1 / 2}, \pm i / w^{1 / 2}$. The points $\pm i w^{1 / 2}$ lie inside the unit circle, while $\pm i / w^{1 / 2}$ lie outside.

Estimate of $\int_{|z|=1} \rho_{1}(z) d z$. Let $\alpha$ be a real positive number such that

$$
\begin{aligned}
\alpha<\inf _{u \in C} \frac{1}{2}\left|w^{1 / 2}\right| & =\frac{1}{2}\left(\frac{1}{x}-\frac{1}{2}\right)^{1 / 2} \cdot \\
\left|\int_{|z|=1} \rho_{1}(z) d z\right| & \leqq\left|\int_{|z|=\alpha} \rho_{1}(z) d z\right|+\left|\operatorname{Res} \rho_{1}(z)\right|_{z= \pm i w^{1 / 2}} \\
\left|\operatorname{Res} \rho_{1}(z)\right|_{z=+i w^{1 / 2}} & =\frac{|1-w|^{r+2 s}}{2\left|w^{s}\right|\left|1-w^{2}\right|}, \\
\left|\int_{|z|=\alpha} \rho_{1}(z) d z\right| & \leqq 2 \pi \alpha^{2} \frac{\left(\alpha^{2}+1\right)^{r+2 s}}{\alpha^{2 s}} M_{1}(w)
\end{aligned}
$$

where $M_{1}(w)=\sup _{|z|=\alpha}\left|w z^{4}+\left(1+w^{2}\right) z^{2}+w^{2}\right|^{-1} . M_{1}(w)$ certainly exists since the choice of $\alpha$ assures that $w z^{4}+\left(1+w^{2}\right) z^{2}+w^{2}$ is bounded away from zero for $|z|=\alpha$. Now, fix $\alpha$ so small that $x\left(\alpha^{2}+1\right)<2$.

Estimate of $\int_{|z|=1} \rho_{2}(z) d z$. Let $\beta$ be a real positive number such that

$$
\begin{aligned}
\beta>\sup _{u \in C} 2 /\left|w^{1 / 2}\right| & =\frac{2}{\left(\frac{1}{x}-\frac{1}{2}\right)^{1 / 2}}, \\
\left|\int_{|z|=1} \rho_{2}(z) d z\right| & \leqq\left|\int_{|z|=\beta} \rho_{2}(z) d z\right|+\left|\operatorname{Res} \rho_{2}(z)\right|_{z= \pm i / w^{1 / 2}} \\
\left|\operatorname{Res} \rho_{2}(z)\right|_{z= \pm i / w^{1 / 2}} & =\frac{|1-w|^{r+2 s}}{2|w|^{\mid s}\left|1-w^{2}\right|},
\end{aligned}
$$




$$
\left|\int_{z=\beta} \rho_{2}(z) d z\right| \leqq 2 \pi \beta^{2} \frac{\left(\beta^{2}+1\right)^{r+2 s}}{\beta^{2 r+2 s}} M_{2}(w)
$$

where $M_{2}(w)=\sup _{|z|=\beta}\left|w z^{4}+\left(1+w^{2}\right) z^{2}+w\right|^{-1}$. The choice of $\beta$ assures that $M_{2}(w)$ exists. Now fix $\beta$ so large that $x\left(\beta^{2}+1\right) / \beta^{2}<2$.

We are now ready to give the estimate of $\nu(r, t)$.

$$
|\nu(r, t)| \leqq \int_{C}\left|\frac{e^{u}}{u}\right|\left|1-w^{2}\right|\left|Q_{r}(w)\right||d u|
$$

where

$$
\begin{array}{r}
Q(w) \leqq \sum_{s=0}^{\infty} \frac{1}{s !(r+s) ! 2^{r+2 s}}\left\{\frac{|1-w|^{r+2 s}}{|w|^{s}\left|1-w^{2}\right|}+\pi \frac{\left(\alpha^{2}+1\right)^{r+2 s} M_{1}}{\alpha^{2 s-2}}\right. \\
\left.+2 \pi \frac{\left(\beta^{2}+1\right)^{r+2 s} M_{2}}{\beta^{2 r+2 s-2}}\right\} .
\end{array}
$$

The estimate (2.6) enables us to write

$$
\begin{aligned}
& \sum_{1}^{\infty}|g(r)||\nu(r, t)| \\
& \quad=\int_{C}\left|\frac{e^{u}}{u}\right|\left|1-w^{2}\right|\left\{\sum_{1}^{\infty}|g(r)|\left|Q_{r}(w)\right|\right\} d u .
\end{aligned}
$$

The proof is now completed by breaking (2.7) into three parts corresponding to the three sums in (2.6). Call these parts $T_{1}, T_{2}$, and $T_{3}$. We give the details showing $T_{1} \rightarrow 0$ as $t \rightarrow 0+$. The details for $T_{2}$ and $T_{3}$ are similar, and somewhat simpler after observing that $M_{1}(w)$ and $M_{2}(w)$ are bounded for $u \in C$ (again by the choice of $\alpha$ and $\beta$ ).

$$
\begin{aligned}
T_{1} & =\int_{C}\left|\frac{e^{u}}{u}\right|\left|1-w^{2}\right| \\
& \cdot\left\{\sum_{1}^{\infty}|g(r)| \sum_{s=0}^{\infty} \frac{1}{s !(r+s) ! 2^{r+2 s}} \frac{|1-w|^{r+2 s}}{|w|^{\mid s}\left|1-w^{2}\right|}\right\} d u .
\end{aligned}
$$

Since $(r+s) ! \geqq r ! s !$ and since $2^{2 s}(s !)^{2} \geqq(2 s)$ ! we have

$$
\begin{aligned}
\sum_{s=0}^{\infty} & \frac{1}{s !(r+s) ! 2^{r+2 s}} \frac{|1-w|^{r+2 s}}{|w|^{s}\left|1-w^{2}\right|} \\
& \leqq \frac{|1-w|^{r}}{\left|1-w^{2}\right| r ! 2^{r}} \sum_{s=0}^{\infty} \frac{|1-w|^{2 s}}{\left(|w|^{1 / 2}\right)^{2 s}} \frac{1}{(2 s) !} \leqq \frac{|1-w|^{r}}{\left|1-w^{2}\right| r ! 2^{r}} e^{|1-w| /|w|^{1 / 2}},
\end{aligned}
$$

so that 


$$
\begin{aligned}
& T_{1} \leqq \int_{C}\left|\frac{e^{u}}{u}\right| e^{|1-w| /|w|^{1 / 2}} \sum_{1}^{\infty} g(r) \frac{|1-w|^{r}}{2^{r} r !}|d u| \\
& \leqq\left. A_{1} \int_{C}\left|\frac{e^{u}}{u}\right| e^{\left|u^{-t}-1\right| \cdot|u|}\right|^{t / 2} \frac{\left|1-u^{-t}\right|}{1-x / 2\left|1-u^{-t}\right|}|d u|
\end{aligned}
$$

where $A_{1}$ is a constant.

Break the path $C$ into two parts, $C^{*}$ and $C_{*}$ as shown:

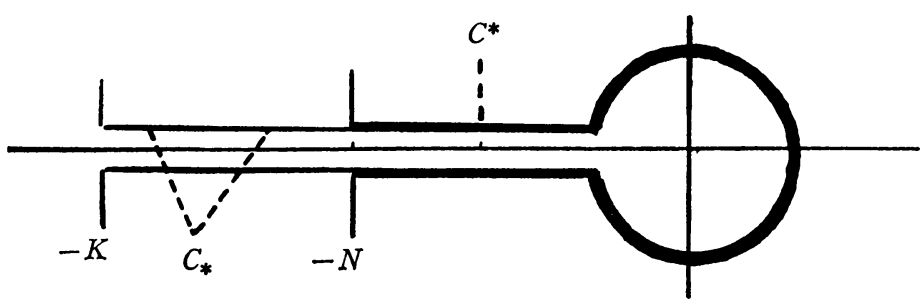

Given $\epsilon>0$ choose $t$ so small that there is a fixed positive number $N<K$ such that

$$
e^{-N} \int_{C_{*}} e^{\left|u^{t}-1\right||u| t / 2} \frac{\left|1-u^{-t}\right|}{1-x / 2\left|1-u^{-t}\right|}|d u|<\frac{\epsilon}{2}
$$

and also

$$
\begin{aligned}
& \sup _{U C^{*}}\left|1-u^{-t}\right|=\left|1-N^{-t}\right| \\
&<\frac{\epsilon}{2}\left(\int_{C^{*}}\left|\frac{e^{u}}{u}\right| e^{\left|u^{t}-1\right|||^{t / 2}} \frac{1}{1-x / 2\left|1-u^{-t}\right|}|d u|\right)^{-1} .
\end{aligned}
$$

This can be done by virtue of the fact that $K \rightarrow+\infty$ as $t \rightarrow 0+$ and the fact that the integrals in (2.8) and (2.9) are seen, by elementary estimates, to be bounded independently of $t$. Thus $T_{1}<\epsilon$, and since $\epsilon$ is arbitrary the assertion follows.

\section{REFERENCES}

1. A. Edrei, On the generating function of a doubly infinite totally positive sequence, Trans. Amer. Math. Soc. vol. 74 (1953) pp. 367-386.

2. G. H. Hardy, Divergent series, Oxford, 1949.

3. I. I. Hirschman and D. V. Widder, The convolution transform, Princeton, 1955.

4. H. Pollard and C. Standish, Inversion of a class of discrete convolution transforms, Scripta Math. vol. 22 (1956) pp. 207-216.

5. G. N. Watson, Theory of Bessel functions, Cambridge, 1922.

6. D. V. Widder, The Laplace transform, Princeton, 1956.

\section{CORNeLl University AND} Michigan State University 